\title{
Phase Diagram from PNJL Models
}

\author{
Kenji Fukushima* \\ Yukawa Institute for Theoretical Physics, Kyoto University, Kyoto 606-8502, Japan \\ E-mail: fuku@yukawa.kyoto-u.ac.jp
}

\begin{abstract}
Possible phase diagrams of QCD at finite temperature and baryon chemical potential are discussed from the point of view of the effective model study especially by means of the Polyakov-loop coupled Nambu-Jona-Lasinio (PNJL) model. Two crossovers associated with quark deconfinement and chiral restoration are located at the (nearly) same temperature as long as the baryon chemical potential is small, but they eventually result in distinct boundaries on the phase diagram with increasing baryon density, which characterizes a region surrounded by two boundary curves. Also, the chiral critical point and the isentropic trajectories around it are critically argued.
\end{abstract}

5th International Workshop on Critical Point and Onset of Deconfinement - CPOD 2009,

June 08 - 122009

Brookhaven National Laboratory, Long Island, New York, USA

\footnotetext{
* Speaker.
} 


\section{Introduction}

There is increasing interest in phase transitions of QCD matter at finite temperature $T$ and baryon density or chemical potential $\mu_{B}$. It has been established that strongly interacting matter undergoes a phase transition at $T$ which is comparable to the QCD energy scale $\Lambda_{\mathrm{QCD}} \sim 200 \mathrm{MeV}$. The phase transition is usually characterized by two approximate order parameters, i.e. the (traced) Polyakov loop $\ell=\frac{1}{3}\langle\operatorname{tr} L\rangle$ and the chiral condensate $\langle\bar{q} q\rangle$. Although they seemingly indicate a common change of the state of QCD matter, $\ell$ and $\langle\bar{q} q\rangle$ belong to completely different dynamics in QCD; the Polyakov loop is a good order parameter for quark deconfinement in the quenched $\left(m_{q} \rightarrow \infty\right)$ limit, while the chiral condensate for chiral restoration in the chiral $\left(m_{q} \rightarrow 0\right)$ limit.

One may wonder that a rapid crossover in one side (quark deconfinement for example) could be a trigger for transitional behavior in the other side (chiral restoration for the same example). This is partially the case indeed. The Polyakov loop and the chiral condensate have a quantum number $0^{++}$same as that of the vacuum, so that they both describe the $0^{++}$glueball and the scalarisoscalar (so-called $\sigma$ ) meson states, which can be mixed up with each other. Because of mixing it is natural to anticipate simultaneous increase or decrease in the order parameters as a function of $T$ and $\mu_{B}$ [1]. This explanation is not really adequate to give a full account for what has been observed in the lattice QCD simulations [3].

In the density region where the Monte-Carlo simulation is feasible it has been found that $\ell$ and $\langle\bar{q} q\rangle$ are monotonically increasing and decreasing, respectively, as $T$ goes from below to above $T_{c}$ which is common to both order parameters. This observation is something more than implied by mixing. In general, only from the mixing argument, we cannot exclude a possibility that there appear two separate crossovers with one dominated by deconfinement and another by chiral restoration. The fact is, however, that quark deconfinement and chiral restoration should take place at the same or nearly same temperature. Two phenomena which are originally opposite to each other with respect to the quark mass must be locked together for a wide range of intermediate quark mass [4].

As attempts to resolve this question about underlying mechanism in order to link quark deconfinement and chiral restoration, a double expansion of strong coupling constant and large dimensions was performed to build an effective model in terms of both order parameters [5, 6, 7, 8]. The strong-coupling model was so successful that it could reproduce qualitative behavior of $\ell$ and $\langle\bar{q} q\rangle$ similar to that resulting from the finite- $T$ lattice QCD simulation. It was not easy, however, for the model to go beyond qualitative agreement and to say anything quantitative, because the model was formulated on the lattice which is far from the continuum limit. Besides, hopping of quarks in the spatial directions is suppressed by the strong coupling constant and all quark excitations are static in configuration space (straight along the temporal direction). The Fermi surface, hence, cannot be formed correctly with a finite $\mu_{B}$ introduced. Moreover, it is hopeless to recover the StefanBoltzmann law at extremely high $T$ due to the presence of dimensional scale other than $T$, that is, a lattice spacing or an ultraviolet (UV) cutoff. By the way the chiral sector of the model obtained in the leading order of the double expansion takes a form of quark interaction through four-fermion vertices. This is reminiscent of a chiral model in the continuum with an ultraviolet cutoff, known as the Nambu-Jona-Lasinio (NJL) model [9,10]. After revisiting the strong-coupling model [7, 8] it had not been so long before an idea was proposed that the NJL model is augmented with the 
Polyakov loop $\ell$ in a way inspired by the strong-coupling form [11]. Such a hybrid model with the Polyakov loop coupling is called the PNJL model [12].

Although the PNJL model aimed to explain the simultaneous crossovers of quark deconfinement and chiral restoration, a bonus has been recognized that thermodynamic quantities such as the pressure, the entropy density, the quark number susceptibility, and so on are in good agreement with those measured in the lattice simulation with dynamical quarks once the model parameters are fixed by the data of the lattice simulation without quarks [12]. Since the lattice QCD simulation is unable to access high- $\mu_{B}$ and low- $T$ regions because of the sign problem, the PNJL model is quite useful there. As we will see later, one important prediction from the PNJL model is that two phase boundaries of deconfinement and chiral crossovers get apart from each other with $\mu_{B}$ comparable to (quantitatively, one third to one half of) the baryon mass [11 14].

When it comes to the phase structure, the existence or location of the chiral critical point of QCD is also of great interest. There, a smooth crossover turns to a second-order critical point, and the phase boundary becomes of first order at higher density than at the critical point [15, 16]. What we can really learn about the critical point from the PNJL model studies is not where the QCD critical point should sit but how easily its existence or location can be affected by small changes in theory [17]. At the moment it is impossible to conclude where or even whether the QCD critical point exists. We must emphasize that this is so because the existence or location of the critical point is extremely sensitive to delicate factors rather than because of model uncertainties. Even though we had a way to come by almost exact answer (for instance lattice simulations in the future when the sign problem is resolved), it would be still difficult to make a precise identification of a second-order critical point distinguished from a steep crossover and from a weak first-order phase transition.

In this article we will discuss the idea of the PNJL model and its setup with model parameters and look into a typical phase diagram from the PNJL model.

\section{Polyakov Loop and Physical Degrees of Freedom}

It is quite nontrivial that the PNJL model can reproduce full QCD thermodynamics around $T_{c}$ because the Polyakov loop is expressed in terms of longitudinal $A_{4}$ (temporal component of the gauge field) and the thermally excited gluons are transverse $A_{i}$. In fact the Polyakov loop is so influential to colored objects that it can control the thermal excitation of transverse gluons as well as quarks. In what follow let us discuss the Polyakov loop coupling with quarks and transverse gluons, respectively.

\subsection{Quarks}

If we assume any interaction effect to be incorporated into the mean-fields (i.e. quasi-particle approximation), the grand-canonical partition function for quasi-quarks is

$$
Z_{\text {quark }}=\prod_{i, p}\left[1+e^{-\left(E_{i}(p)-\mu_{q}\right) / T}\right]\left[1+e^{-\left(E_{i}(p)+\mu_{q}\right) / T}\right],
$$

where $i$ runs over 3 colors, $2 \sim 3$ flavors, and 2 spin states. Instead of $\mu_{B}$ a quark chemical potential $\mu_{q}=\mu_{B} / 3$ is used above. We did not consider the Polyakov loop coupling yet. The Polyakov 
loop, $L$, is a $3 \times 3$ matrix in color space associated with thermal quarks, which represents a colorscreening phase factor in gluonic matter. Therefore, the covariant coupling with the $A_{4}$ field leads to a coupling of $L$ and $L^{\dagger}$ with the thermal Boltzmann factor as

$$
\begin{aligned}
Z_{\text {quark }}= & \prod_{i, p} \operatorname{det}\left[1+L e^{-\left(E_{i}(p)-\mu_{q}\right) / T}\right]\left[1+L^{\dagger} e^{-\left(E_{i}(p)+\mu_{q}\right) / T}\right] \\
= & \prod_{i, p}\left[1+3 \ell e^{-\left(E_{i}-\mu_{q}\right) / T}+3 \bar{\ell} e^{-2\left(E_{i}-\mu_{q}\right) / T}+e^{-3\left(E_{i}-\mu_{q}\right) / T}\right] \\
& \times\left[1+3 \bar{\ell} e^{-\left(E_{i}+\mu_{q}\right) / T}+3 \ell e^{-2\left(E_{i}+\mu_{q}\right) / T}+e^{-3\left(E_{i}+\mu_{q}\right) / T}\right] .
\end{aligned}
$$

Here we note that $\ell$ denotes the traced Polyakov loop $\ell=\frac{1}{3} \operatorname{tr} L$ and $\bar{\ell}$ the anti-Polyakov loop $\bar{\ell}=$ $\frac{1}{3} \operatorname{tr} L^{\dagger}$. At zero baryon density $\bar{\ell}$ is just identical with $\ell$, but once a finite density is turned on, $\bar{\ell}>\ell$ for positive $\mu_{q}$ (or $\mu_{B}$ ) because an antiquark is more efficiently screened in a medium with quarks. Each term has a clear physical meaning in eq. 2.2 ; ; in the angle brackets the first term without the Boltzmann factor represents no particle excitation, the second term proportional to $e^{-\left(E_{i}-\mu_{q}\right) / T}$ a single particle excitation , the third term proportional to $e^{-2\left(E_{i}-\mu_{q}\right) / T}$ a double particle or a diquarklike excitation in the color antitriplet channel, and the last term a colorless baryon-like excitation.

Just for demonstrating how the Polyakov loop coupling controls thermal excitations we shall compute the quark pressure $p_{\text {quark }}=T V^{-1} \ln Z_{\text {quark }}$ from the partition function 2.2) in which the quark mass and chemical potential are set to be zero so that the pressure is simply proportional to $T^{4}$. Then, in the free-quark limit (i.e. $\ell=\bar{\ell}=1$ ), the Stefan-Boltzmann law reads $p_{\text {free }}=\left(63 \pi^{2} / 180\right) T^{4}$ for massless three flavors. We measure the quark pressure in the unit of the Stefan-Boltzmann value; $v=p_{\text {quark }} / p_{\text {free }}$, which actually counts the relevant degrees of freedom. We plot $v$ as a function of $\ell$ in the simple case with $m_{q}=\mu_{q}=0$ as is presented in the left of fig. 1 It is apparent that the pressure contribution is nonzero but nearly vanishing at $\ell=0$ and is almost linearly increasing as $\ell$ becomes larger. If we recall that the Polyakov loop is an order parameter for quark deconfinement, we can naturally understand this; no pressure from quarks in the confined phase at $\ell=0$ and the free-quark limit is reached in the deconfined phase at $\ell=1$. It should be mentioned here that the colorless baryon-like term in eq. (2.2) remains even at $\ell=0$ but its contribution to the pressure is suppressed by a factor $1 / 3^{4}=0.012$ as compared to the free-quark excitation.

\subsection{Transverse gluons}

From the theoretical argument based on global (center) symmetry in the pure gluonic sector the traced Polyakov loop in the color fundamental representation serves as an order parameter for quark deconfinement but not for gluon deconfinement. Because gluons can screen other gluons, a long-range confining force between gluons is saturated by string breaking due to two glueball excitations even when color charge is strictly confined. Thus, it is not clear $a$ priori whether $\ell$ can be useful to parametrize the pressure stemming from transverse gluons.

In the same manner as in the previous case of quarks we can consider the grand-canonical partition function for quasi-gluons, that is,

$$
Z_{\mathrm{gluon}}=\prod_{i, p} \operatorname{det}\left[1-L_{\mathrm{adj}} e^{-p / T}\right]
$$



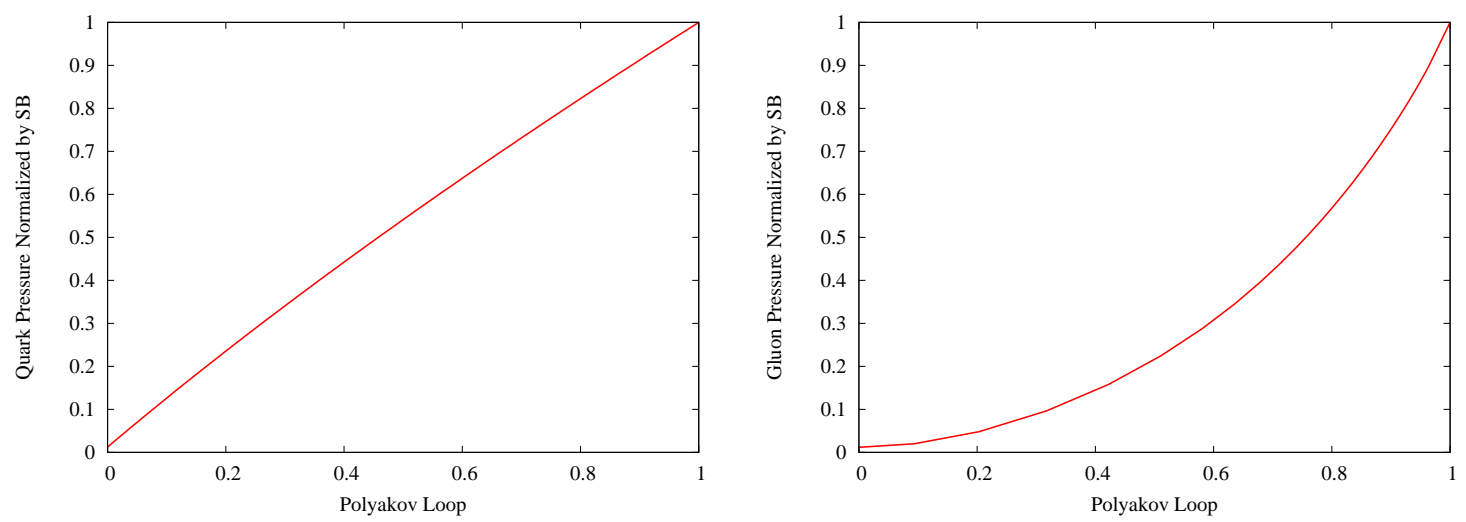

Figure 1: Pressure contributions from quasi-quarks (left) and quasi-gluons (right) as a function of the traced Polyakov loop $\ell$ in the color fundamental representation.

where $i$ runs over 8 colors and 2 transverse polarizations. Here we introduced $L_{\text {adj }}$ to denote an $8 \times 8$ Polyakov loop matrix in the color adjoint representation. In the Stefan-Boltzmann limit with $L_{\text {adj }}=1$, the pressure is $p_{\text {free }}=\left(16 \pi^{2} / 90\right) T^{4}$. Again, the gluon pressure is calculated from eq. 2.3 in the unit of the Stefan-Boltzmann value and the numerical result is shown in the right of fig. 1 , The pressure dependence on $\ell$ is not linear this time but almost quadratic reflecting the fact that the adjoint Polyakov loop is expressed as $\left\{L_{\mathrm{adj}}\right\}_{a b}=2 \operatorname{tr}\left[t_{a} L t_{b} L^{\dagger}\right]$ in terms of the fundamental one.

It might be appropriate here to explain some more details on the evaluation of the transverse pressure. Unlike the quark contribution (2.2) we cannot rewrite the gluon pressure 2.3) only in terms of $\ell$ and $\bar{\ell}$. It requires us, therefore, to adopt an elaborated procedure for the mean-field approximation 15,7, 18, 19, 20, 21]. Then, a mean-field weight factor is introduced as $e^{-S_{\mathrm{mf}}}=\prod e^{x \mathrm{tr} L}$, so that the expectation value of the fundamental Polyakov loop, $\ell(x)=\int d L \frac{1}{3} \operatorname{tr} L e^{x \operatorname{tr} L} / \int d L e^{x \operatorname{tr} L}$ takes a nonzero value proportional to a mean-field variable $x$ (that is; $x$ is a source conjugate to $\ell$ ). In the same way the gluon pressure is numerically calculated as a function of $x$, from which we can eliminate the $x$-dependence using $\ell=\ell(x)$.

It is surprising that a Polyakov loop value as large as 0.8 yields a transverse pressure less than 0.6 times the Stefan-Boltzmann value. The right of fig. 1 manifests the importance of the Polyakov loop even in the deconfined region where $\ell$ is substantially large but do not yet reach unity. In this region the screening effect by the Polyakov loop overwhelmingly governs the degrees of freedom allowed in the system, which would cause nonperturbative deviations from the StefanBoltzmann limit however weakly the matter interacts itself. Such a highly nontrivial state of matter is sometimes referred to as a semi-quark-gluon plasma (semi-QGP) [22].

\section{Thermodynamics in Pure Gluodynamics}

So far we have seen that the Polyakov loop can control the thermal excitation even for transverse gluons which couple with the adjoint Polyakov loop which is always nonzero due to color screening by other gluons. It should be acceptable to parametrize the physical pressure only in terms of $\ell$, which can be justified by the observation in the right of fig. 1 that $\ell$ is practically an order parameter for gluon deconfinement as well. 

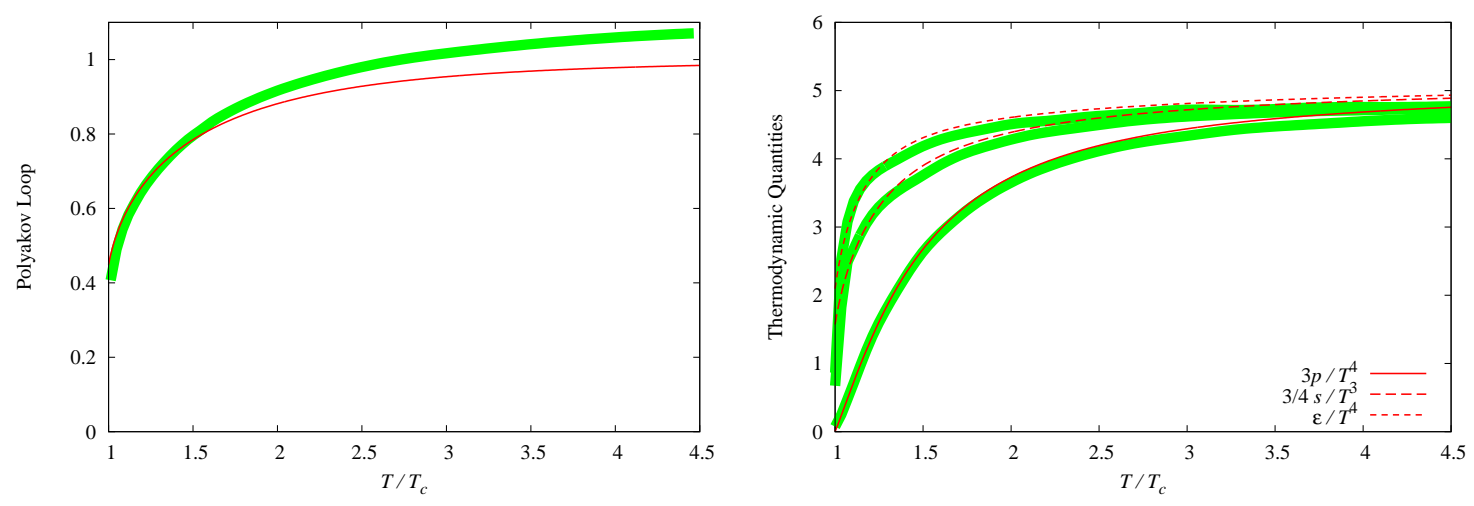

Figure 2: Comparison between the quenched lattice data (green thick curves) and the results from the potential ansatz (red thin curves). The left shows the Polyakov loop and the right shows the thermodynamic quantities.

Therefore, we may well assume that the pressure in pure gluodynamics is a function of $\ell$ with coefficients depending on $T$. A frequently used ansatz [23] is

$$
V(\ell)=-\frac{1}{2} a(T) \ell \bar{\ell}+b(T) \ln \left[1-6 \ell \bar{\ell}+4\left(\ell^{3}+\bar{\ell}^{3}\right)-3(\ell \bar{\ell})^{2}\right]
$$

where $a(T) / T^{4}=\left(3.51-2.47 t^{-1}+15.2 t^{-2}\right)$ and $b(T) / T^{4}=-1.75 t^{-3}$ with $t=T / T_{c}$. There are three independent parameters in this ansatz because of a constraint that $V(\ell=1)$ should obey the Stefan-Boltzmann law. This form (3.1) is very similar to another ansatz with two parameters $\alpha$ and $\beta$ motivated by the strong coupling analysis; $a(T) / T=54 \beta e^{-\alpha / T}$ and $b(T) / T=\beta$, where $\beta$ has mass dimension 3 whose scale comes from the UV cutoff [11,17]. In the vicinity of $T_{c}$ in any case both parametrizations end up with approximately same thermodynamics.

From the stationary condition $d V / d \ell=0$ the Polyakov loop expectation value is extracted from eq. 3.1], which is compared with the lattice date taken from Ref. [24] as presented in the left of fig. 2 Because of renormalization effect the Polyakov loop in the lattice simulation exceeds unity, while $\ell$ never gets greater than unity. This is why the agreement looks worse at high temperature. We see, in contrast, that the fitting works nicely and the potential reproduces the thermodynamic quantities such as the pressure $p$, the entropy density $s$, and the internal energy density $\varepsilon$ well. For the sake of comparison we plot the output from eq. (3.1) and the the lattice data taken from Ref. [25]. Now we have finished fixing the pure gluonic sector.

\section{Thermodynamics with Dynamical Quarks}

The quark sector is described by the NJL model which has three parameters, namely, the current quark mass $m_{u}=m_{d}$, the four-fermion coupling constant $g_{s}$, and the UV cutoff $\Lambda$ for the two-flavor case, and two more parameters, namely, the strange quark mass $m_{s}$ and the 't Hooft interaction strength $g_{d}$ for the three-flavor case. The model parameters are fixed by the hadron properties; $m_{\pi}, f_{\pi}$, and $\langle\bar{q} q\rangle$ for two-flavor matter, and $m_{K}$ and $m_{\eta^{\prime}}$ in addition for three-flavor matter. 

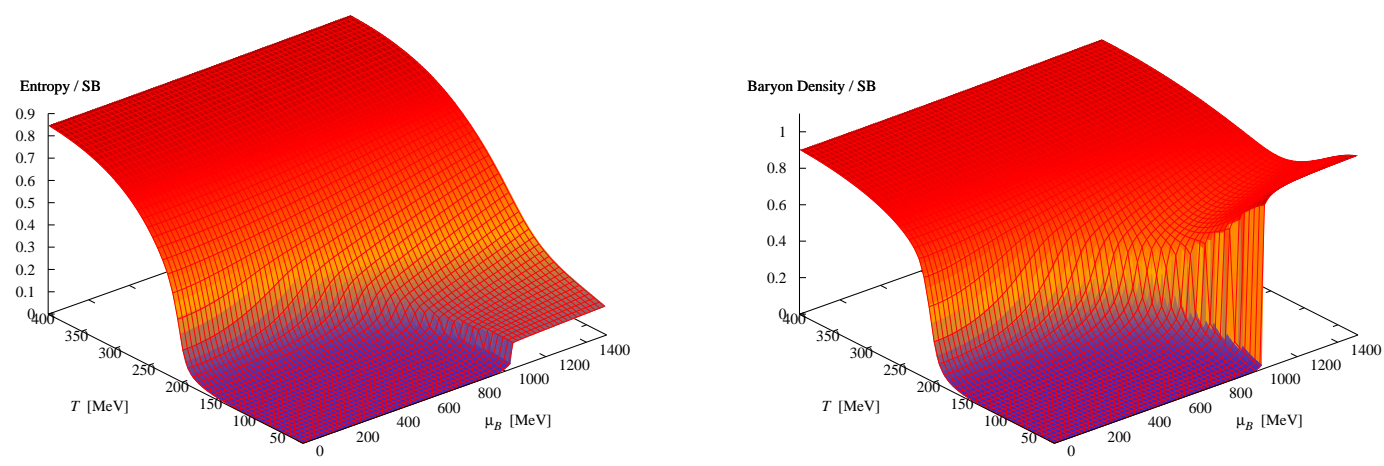

Figure 3: Entropy density (left) and baryon number density (right) normalized by the Stefan-Boltzmann limit values as functions of $T$ and $\mu_{B}$ obtained in the three-flavor PNJL model.

The NJL model with the Polyakov loop coupling in eq. (2.2) and the potential in eq. (3.1) gives full thermodynamics in the plane spanned by $T$ and $\mu_{B}$. We plot some of thermodynamic quantities in fig. 3. The left shows the entropy density that is $s=-\partial \Omega / \partial T$ and the right shows the baryon number density that is $n_{B}=-\partial \Omega / \partial \mu_{B}$. The reason why we chose them is that $s$ and $n_{B}$ should be suitable for detecting a change of the state of matter as $T$ and $\mu_{B}$ increases, respectively [26]. One can see that $s$ is an increasing function of $T$ whose dependence on $\mu_{B}$ is mild, while $n_{B}$ jumps drastically with increasing $\mu_{B}$. One possible interpretation is that $s$ carries information on the deconfinement and $n_{B}$ is an effective order parameter for the realization of so-called quarkyonic matter [27, 28, 29].

\section{Phase Diagram}

The PNJL model is capable of dealing with $\ell$ and $\langle\bar{q} q\rangle$ both as $T$ and $\mu_{B}$ change, from which the phase diagram is deduced. The phase diagram in the context of the PNJL model was first depicted in Ref. [23], and in Ref. [14] it was clearly recognized that the deconfinement line characterized by the Polyakov loop (susceptibility) becomes distinct from the chiral transition line characterized by the chiral condensate (or susceptibility). The model has been extended to the three-flavor case later on 3031 17. In the presence of the Polyakov loop background it is not easy to take account of the diquark condensate. In Refs. [23, 32, 33] the diquark condensate has been considered in a gauge dependent treatment, but as pointed out in Ref. [32] such a treatment breaks color neutrality even in normal quark matter, which is unphysical artifact. A remedy is formulated in Ref. [21], but for technical reasons its application to the color superconducting phase has not been done yet.

So far, in view of existing works on the phase diagram using the PNJL model [23, 14, 30, 31, 17] the robust prediction is that two separate phase boundaries appear on the phase diagram. A typical example is shown in fig. 4 .

This phase diagram is topologically the same as suggested by the large $N_{c}$ argument 27,28, 29]. In the region surrounded by two phase boundaries (which have finite width in fig. 4 because they are both crossovers) the color degrees of freedom is confined because of $\ell \simeq 0$ and chiral symmetry is restored and the Fermi sphere is filled by light quarks. Intuitive understanding on this 


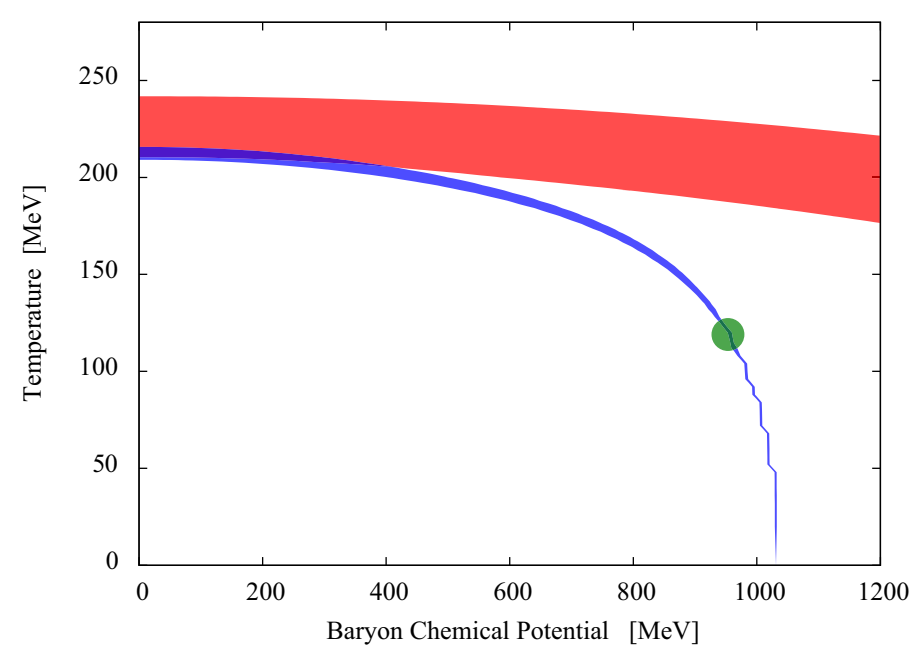

Figure 4: Typical phase diagram inferred from the PNJL model. The red band indicates a region in which $\ell$ takes a value from 0.4 to 0.6 and the blue band a region in which $\langle\bar{q} q\rangle$ from 0.4 to 0.6 . A circle locates the critical point below which the chiral phase transition is of first order and the blue band has no width.

peculiar state is as follows; the degenerated Fermi matter consists of light quarks and any excitation on top of the Fermi surface should be colorless like mesons and baryons.

The chiral critical point is indicated by a circle on fig. 4. The location or even existence of this point is easily changed by many factors, however. The lattice QCD simulation results in a significant drop in the topological susceptibility at $T_{c}$, which suggests strong suppression of the topological excitation above $T_{c}$, and thus a reduction of the 't Hooft interaction which is proportional to the instanton density. The effect of $\mathrm{U}(1)_{\mathrm{A}}$ restoration on the location of the critical point has been investigated in Refs. [30, 17] by means of the PNJL model and it has been found that only $35 \%$ reduction of the 't Hooft coupling constant is enough to wash the critical point away from the phase diagram. Also, at finite density, a renormalization of the chemical potential by the vectorvector interaction is expected, as considered already in the earliest work [15]. The effect of the vector interaction has been discussed in the context of the QCD phase diagram [14, 34, 17, 35, 36]. A vector-vector coupling constant as small as only $10 \sim 20 \%$ of the four-fermion coupling constant in the scalar channel is enough to make the critical point disappear from the phase diagram. Of course we cannot say that the chiral critical point does not exist from these analysis, but we should keep in mind that locating the critical point suffers from delicate subtleties. In the lattice simulation in the staggered fermion the $\mathrm{U}(1)_{\mathrm{A}}$ anomaly might not be fully recovered and so, even without the sign problem, a reliable determination of the critical point location is challenging. We could learn this lesson from the model analysis, and, though the model does not give us a final theory answer, the model is very useful as long as one treats the results with due respect.

Finally let us take a quick look at the isentropic trajectories [37 38]. The advantage of the PNJL model is, as stated before, that it can describe not a part of but full thermodynamics. Therefore, the isentropic trajectories defined by a line on which the entropy to baryon number ratio $s / n_{B}$ is constant should be approximated better than any other chiral models [39, 40]. It is notable that in any model studies [37,38, 39, 40] no special behavior around the critical point has been found. 


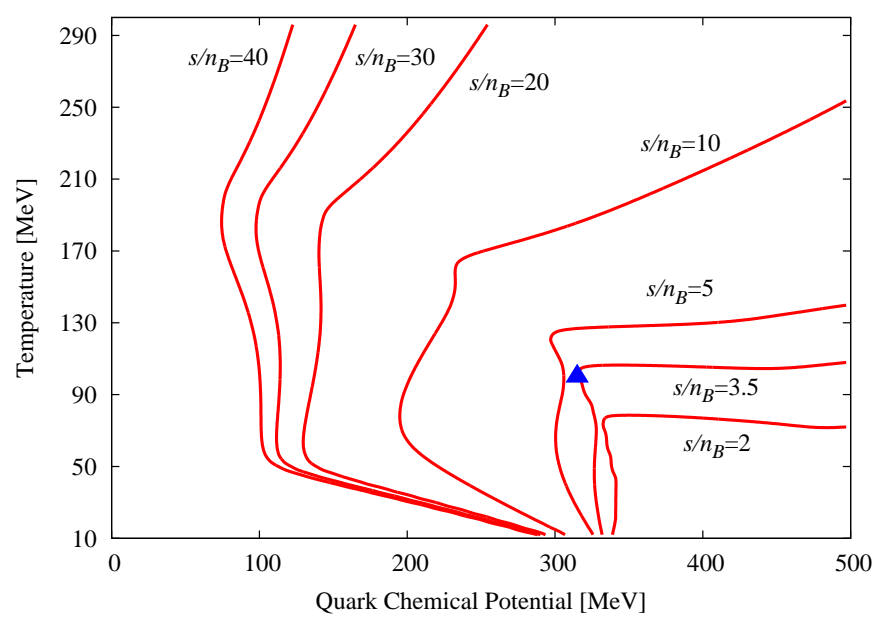

Figure 5: Isentropic trajectories on the phase diagram taken from Ref. 38]. In the above figure the horizontal axis is not the baryon chemical potential $\mu_{B}$ but the quark chemical potential $\mu_{q}$ which is one third of $\mu_{B}$.

Figure 5]shows an example taken from Ref. [38] in which the three-flavor PNJL model is employed. There are two reasons for this; one is that the critical region associated with the chiral critical point is not wide on the $\mu_{B}-T$ plane, probably around $10 \mathrm{MeV}$ or so in both $T$ and $\mu_{B}$. The other is that the critical part in the free energy does not diverge and thus $s / n_{B}$ is never singular at the critical point. It is most unlikely that a focusing effect eases to pinpoint the chiral critical point in collider experiments. We should not take the mode results like figs. 4and5 too seriously, and nevertheless, the model results come to us with an impression that we must rely on tremendous luck to settle the critical point business down in real experiments.

\section{References}

[1] S. Digal, E. Laermann and H. Satz, Eur. Phys. J. C 18, 583 (2001).

[2] A. Mocsy, F. Sannino and K. Tuominen, Phys. Rev. Lett. 92, 182302 (2004).

[3] Y. Hatta and K. Fukushima, Phys. Rev. D 69, 097502 (2004); arXiv:hep-ph/0311267.

[4] F. Karsch, E. Laermann and A. Peikert, Nucl. Phys. B 605, 579 (2001).

[5] A. Gocksch and M. Ogilvie, Phys. Rev. D 31, 877 (1985).

[6] E. M. Ilgenfritz and J. Kripfganz, Z. Phys. C 29, 79 (1985).

[7] K. Fukushima, Phys. Lett. B 553, 38 (2003); Phys. Rev. D 68, 045004 (2003).

[8] K. Fukushima, Prog. Theor. Phys. Suppl. 153, 204 (2004).

[9] S. P. Klevansky, Rev. Mod. Phys. 64, 649 (1992).

[10] T. Hatsuda and T. Kunihiro, Phys. Rept. 247, 221 (1994).

[11] K. Fukushima, Phys. Lett. B 591, 277 (2004).

[12] C. Ratti, M. A. Thaler and W. Weise, Phys. Rev. D 73, 014019 (2006). 
[13] C. Ratti, S. Roessner and W. Weise, Phys. Lett. B 649, 57 (2007).

[14] C. Sasaki, B. Friman and K. Redlich, Phys. Rev. D 75, 074013 (2007).

[15] M. Asakawa and K. Yazaki, Nucl. Phys. A 504, 668 (1989).

[16] A. Barducci, R. Casalbuoni, S. De Curtis, R. Gatto and G. Pettini, Phys. Lett. B 231, 463 (1989); A. Barducci, R. Casalbuoni, G. Pettini and R. Gatto, Phys. Rev. D 49, 426 (1994).

[17] K. Fukushima, Phys. Rev. D 77, 114028 (2008) [Erratum-ibid. D 78, 039902 (2008)].

[18] E. Megias, E. Ruiz Arriola and L. L. Salcedo, Phys. Rev. D 74, 065005 (2006).

[19] K. Fukushima and Y. Hidaka, Phys. Rev. D 75, 036002 (2007).

[20] H. M. Tsai and B. Muller, J. Phys. G 36, 075101 (2009).

[21] H. Abuki and K. Fukushima, Phys. Lett. B 676, 57 (2009).

[22] Y. Hidaka and R. D. Pisarski, Phys. Rev. D 78, 071501 (2008).

[23] S. Roessner, C. Ratti and W. Weise, Phys. Rev. D 75, 034007 (2007).

[24] S. Gupta, K. Huebner and O. Kaczmarek, Phys. Rev. D 77, 034503 (2008).

[25] G. Boyd, J. Engels, F. Karsch, E. Laermann, C. Legeland, M. Lutgemeier and B. Petersson, Nucl. Phys. B 469, 419 (1996).

[26] Y. Hidaka and K. Fukushima, work under completion.

[27] L. McLerran and R. D. Pisarski, Nucl. Phys. A 796, 83 (2007).

[28] Y. Hidaka, L. D. McLerran and R. D. Pisarski, Nucl. Phys. A 808, 117 (2008).

[29] L. McLerran, K. Redlich and C. Sasaki, Nucl. Phys. A 824, 86 (2009).

[30] W. j. Fu, Z. Zhang and Y. x. Liu, Phys. Rev. D 77, 014006 (2008).

[31] M. Ciminale, R. Gatto, N. D. Ippolito, G. Nardulli and M. Ruggieri, Phys. Rev. D 77, 054023 (2008).

[32] H. Abuki, M. Ciminale, R. Gatto, G. Nardulli and M. Ruggieri, Phys. Rev. D 77, 074018 (2008).

[33] D. Gomez Dumm, D. B. Blaschke, A. G. Grunfeld and N. N. Scoccola, Phys. Rev. D 78, 114021 (2008).

[34] M. Kitazawa, T. Koide, T. Kunihiro and Y. Nemoto, Prog. Theor. Phys. 108, 929 (2002).

[35] K. Fukushima, Phys. Rev. D 78, 114019 (2008).

[36] Z. Zhang and T. Kunihiro, Phys. Rev. D 80, 014015 (2009).

[37] T. Kahara and K. Tuominen, Phys. Rev. D 78, 034015 (2008).

[38] K. Fukushima, Phys. Rev. D 79, 074015 (2009).

[39] O. Scavenius, A. Mocsy, I. N. Mishustin and D. H. Rischke, Phys. Rev. C 64, 045202 (2001).

[40] E. Nakano, B. J. Schaefer, B. Stokic, B. Friman and K. Redlich, arXiv:0907.1344 [hep-ph]. 